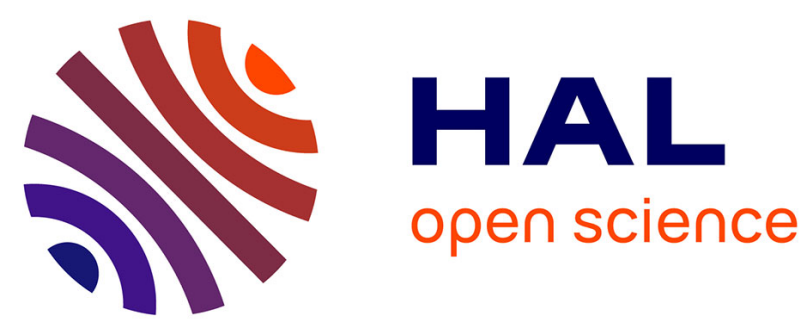

\title{
A theoretical model for the prediction of initial growth angles and sites of fretting fatigue cracks
}

\author{
Valérie Lamacq, Marie-Christine Baietto, Léo Vincent
}

\section{To cite this version:}

Valérie Lamacq, Marie-Christine Baietto, Léo Vincent. A theoretical model for the prediction of initial growth angles and sites of fretting fatigue cracks. Tribology International, 1997, 30 (6), pp.391-400. 10.1016/S0301-679X(96)00068-0 . hal-01951926

\section{HAL Id: hal-01951926 \\ https://hal.science/hal-01951926}

Submitted on 7 Jul 2021

HAL is a multi-disciplinary open access archive for the deposit and dissemination of scientific research documents, whether they are published or not. The documents may come from teaching and research institutions in France or abroad, or from public or private research centers.
L'archive ouverte pluridisciplinaire HAL, est destinée au dépôt et à la diffusion de documents scientifiques de niveau recherche, publiés ou non, émanant des établissements d'enseignement et de recherche français ou étrangers, des laboratoires publics ou privés. 


\title{
A theoretical model for the prediction of initial growth angles and sites of fretting fatigue cracks
}

\author{
Valérie Lamacq*, Marie-Christine Dubourg* and Léo Vincent ${ }^{\dagger}$
}

The crack initiation mechanisms under fretting fatigue conditions are investigated. Fretting fatigue tests have been conducted on an aluminium alloy. Two major crack initiation mechanisms are experimentally observed. They are theoretically identified by employing a simple dislocation dipole model and taking into consideration the reverse sliding along dislocation layers and the influence of the distance between these layers. It is shown that cracks may initiate either by an extrusion-intrusion mechanism or a fatigue tensile process. Two parameters respectively linked to each of these initiation mechanisms are proposed to predict crack location. The theoretical crack initial growth directions and locations are shown to correlate very well with the experimental ones. With regards to these initiation processes, a spall detachment mechanism is proposed considering the interaction of microcracks.

Keywords: fretting fatigue, crack initiation model, spall detachment

\section{Introduction}

The aim of this study is to identify parameters governing crack initiation and thus to predict initial crack growth angles and locations, under fretting fatigue loading.

Three questions are generally asked when studying the crack initiation process:

- Where will the crack nucleate?

- How long will this process take?

- Along which direction will the initial growth take place?

To answer these questions three types of studies are conducted on crack initiation. The first ones aim at predicting where initiation might occur. Dang $\operatorname{Van}^{1}$ proposed a criterion considering the elasto-plastic behaviour of materials to predict initiation sites. This

*Laboratoire de Mécanique des Contact, UMR CNRS 5514, INSA de Lon, 20 A v.A.E instein, $69621 \mathrm{~V}$ illeurbanne $C$ edex, France

Dépirtement Matériaux Mécanique Physique, CNRS URA 447. Ecole Centrale de Lyon, 36 Av. Guy de Collongue, BP 163, 69131 Ecullv: Cedex.France criterion has the advantage of being easily applicable to multiaxial stress fields. Ruiz et al. ${ }^{2}$ conducted experimental investigations into fretting in the dovetail roots of a turbine blade. They proposed two parameters to estimate the surface damage which depends on the work done by the friction force. The fretting damage was thus quantified through the parameter $\tau . \delta$ : the product of the shear stress and the relative slip between the surfaces in contact. They related the location of a crack to a stress/fretting damage parameter: $\sigma . \tau . \delta, \sigma$ being the tangential tensile stress. This parameter was also used to estimate the fatigue life during their experiments. Hills and Nowell $^{3}$ tested this criterion under fretting fatigue conditions. They showed that initiation was likely to appear close to the trailing edge of the contact, where a sharp peak of the latter parameters was reached. Lamacq et al ${ }^{4}$ related the variation of $\sigma_{x x}$ to crack initiation and compared the theoretical initiation sites to experimental ones. Here again, it was shown that cracks would nucleate near the edge of the contact in the fretted area. Actually, all the proposed parameters predict crack initiation under fretting fatigue conditions at roughly the same location.

The second approach aims at predicting the number of cycles to initiation. Mura and Nakasone ${ }^{5}$ proposed a 
theory to provide $\mathrm{S}-\mathrm{N}$ curves for crack initiation. They considered the dependence of crack initiation on material parameters such as yield strength and grain size. - Here, dislocation motion and energy dissipation were taken into account. They showed that the smaller the grain size, the higher the fatigue strength for crack initiation. Cheng et al. $^{6}$ considered dislocation pile-up to predict crack initiation life under contact fatigue. The influence of residual stress, hardness, temperature and irreversibility of plastic deformation was taken into consideration. All these approaches are in good agreement with experimental results.

The third approach aims at predicting the crack initiation direction. Few studies were carried out on crack initiation direction under fretting fatigue conditions. Nowell et $a l^{7}$ considered the variation of the stress intensity factors to study each crack growth stage under these loading conditions. But the applicability of the usual stress intensity factor expressions developed for long cracks, to very short cracks existing in the early growth stage is very questionable, the validity of LEFM for such crack lengths being under question. Actually, it may not be appropriate to study stress intensity factor variations to predict the direction of a crack that still does not exist! On the other hand, Yamashita and Mura ${ }^{8}$ considering the stresses influencing dislocation motion, predicted crack initiation under repeated oblique force. They also considered the influence of a contact asperity in order to understand the mechanism of the initiation of experimentally observed pitting cracks. This method, in taking into consideration a realistic initiation mechanism, seems more appropriate for initial crack growth direction prediction.

Considering the movements of dislocations, the crack initiation mechanism will here be identified either as a process of extrusion and intrusion of slip bands as described by Cottrell or Mott or as a tensile fatigue process. Once the two initiation mechanisms are theoretically identified, two specific parameters are linked respectively to each mechanism to predict the crack initiation sites. The model proposed here is thus able to predict initial crack growth directions and locations taking into consideration a physical phenomenon. As experimentally observed, a large number of microcracks are initiated in the fretted region near the edge of the contact as well as in the fretted region. It will be shown that the interaction of the microcracks initiated through these two crack initiation mechanisms may lead to spall detachment.

\section{Experiments and observations}

The experimental results presented are concerned with fatigue crack initiation under fretting wear conditions. A more general campaign on cracking behaviour of three aeronautical aluminium alloys under fretting conditions has been conducted and presented more extensively elsewhere ${ }^{4.9}$. This general study was undertaken in order to analyse the cracking phenomena under cumulative effects of contact loading and external static traction. In the following text, 'fretting fatigue' is used to describe these loading conditions. The main experimental observations on crack initiation obtained on 7075 aluminium alloy will be briefly presented.

\section{Experimental procedure and conditions}

The apparatus is based on the fretting wear device developed at the Ecole Centrale de Lyon'. This fretting wear device was superimposed on a static loading frame. A full description of the fretting fatigue apparatus is reported elsewhere ${ }^{9}$. A brief description of the apparatus is shown in Fig. 1(a).

A spherical aluminium alloy piece (labelled 2 in Fig. $\mathrm{l}(\mathrm{a})$ ) is pressed against the planar tested sample (labelled 1 in Fig. 1(a)) of the same alloy. This normal force, $F_{n}$, remains constant during the entire test. The spherical body is submitted to a reciprocating microdisplacement, $\delta$, through a piston ring (labelled 3 in Fig. l(a)) and thus rubs against the planar specimen. This micro-displacement produced on the surface induces an oscillating tangential force, $Q(t)$, in the contact area. The planar piece also undergoes a static uniaxial traction, $\sigma_{\mathrm{D}}$. The experimental conditions considered for this study and the mechanical properties of the aluminium alloy tested are summarized in Table 1.

\section{Fretting contact morphology}

In the present study, the contact conditions considered are related to a mixed regime next to sticking. Fatigue cracks are the main surface degradation observed under these loading conditions. The contact zone is divided into two distinct domains (Fig. 1 (b)). The central zone of the contact is a disk encircled by an annulus whose boundary is blurred. The inner central zone corresponds to the stick zone. Fretting damage is not significant in this domain. The outer annulus corresponds to a microsliding zone. The micro-sliding condition is more damaging for the surface than in the stick zone, more wear damage being involved during sliding. The particle loss is facilitated by the sliding displacement, unlike in the stick zone where particles are locked.

No significant plastic deformation is observed at the specimen surface at the end of tests. The depth of deterioration in the sliding zone due to spall detachment is less than $10 \mu \mathrm{m}$.

\section{Experimental observations on fretting cracks}

Multiple cracks initiated in the contact zone. Only two of them, situated symmetrically from the centre of the contact, propagated whilst all the others were self arrested. On the plane specimen surface, $(x 0 z$, in Fig. (b)) the two cracks propagated perpendicularly to the fretting direction, with a semi-elliptical shape. They join each other at each edge of the specimen, as observed in Fig. 1(b).

Looking at cross-section ( $x 0 y$ ) (Fig. 3), these multiple cracks clearly appear. All along the experiments, cracks were observed to initiate along two major distinct directions:

- The first crack initiation type corresponds to an initial cracking at a low angle with respect to the specimen surface. Cracks are then observed to grow initially along a direction of approximately $25^{\circ}$ as observed in Fig. 2(a). This type of crack was detected in the fretted annulus of the contact area. 


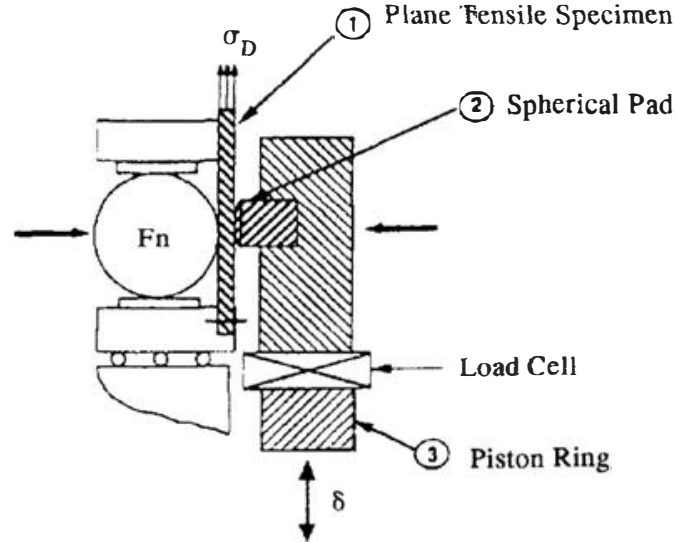

(a)

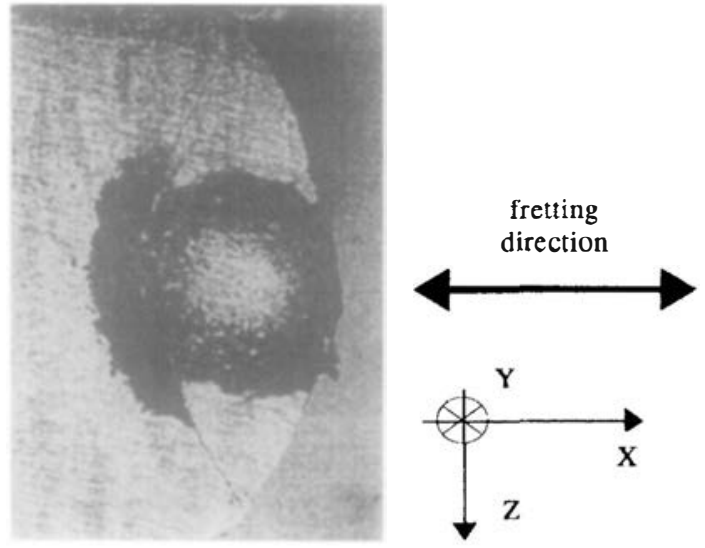

(b)

Fig. 1 (a) Experimental apparatus; (b) view of the specimen surface contact

Table 1 Mechanical properties and experimental conditions

\begin{tabular}{ll} 
Mechanical properties & $\begin{array}{l}\text { Experimental conditions } \\
\text { Amplitude of } \\
\text { displacement, } \delta: \\
\pm 35 \mu \mathrm{m}\end{array}$ \\
$\begin{array}{l}\text { Fluminium alloy: } \\
7075\end{array}$ & $\begin{array}{l}\text { Normal foncy: } 5 \mathrm{~Hz} \\
\text { Young's modulus: }\end{array}$ \\
$\begin{array}{l}72000 \mathrm{MPa} \\
\text { Poisson's ratio: } 0.3\end{array}$ & $\begin{array}{l}\text { Static uniaxial traction: } \\
230 \mathrm{MPa} \\
\text { Radius of spherical } \\
\text { sample: } 0.3 \mathrm{~m}\end{array}$ \\
\hline
\end{tabular}

- The second crack initiation type corresponds to an initial cracking at a large angle with respect to the ccmponent surface. Cracks are then observed to grow initially along a direction approximately perpendicular to the surface. This is observed in Fig. 2(b). This type of crack generally appears near the edge of the contact area, inside or outside this domain.

A crack network also exists in the opposing spherical specimen (Fig. 3). This counterface is only submitted to fretting loading (fretting wear). The two longest cracks of this piece are located opposite to the two principal cracks of the plane specimen. These fretting wear cracks have the same locations and inclinations as the fretting fatigue cracks. They are shorter than the latter ones. Figure 3 presents the crack network existing in a planar specimen and the crack network observed in its opposing sphere. Multiple cracks are observed in both pieces. The two crack initiation directions defined previously can be observed on these two figures.

\section{Theoretical approach to fretting fatigue crack initiation mechanisms}

During the experiments, crack initiation first appears in the meridian plane of the contact area (in Fig. 1(b) and schematically in Fig. 4(a)). The early initiation

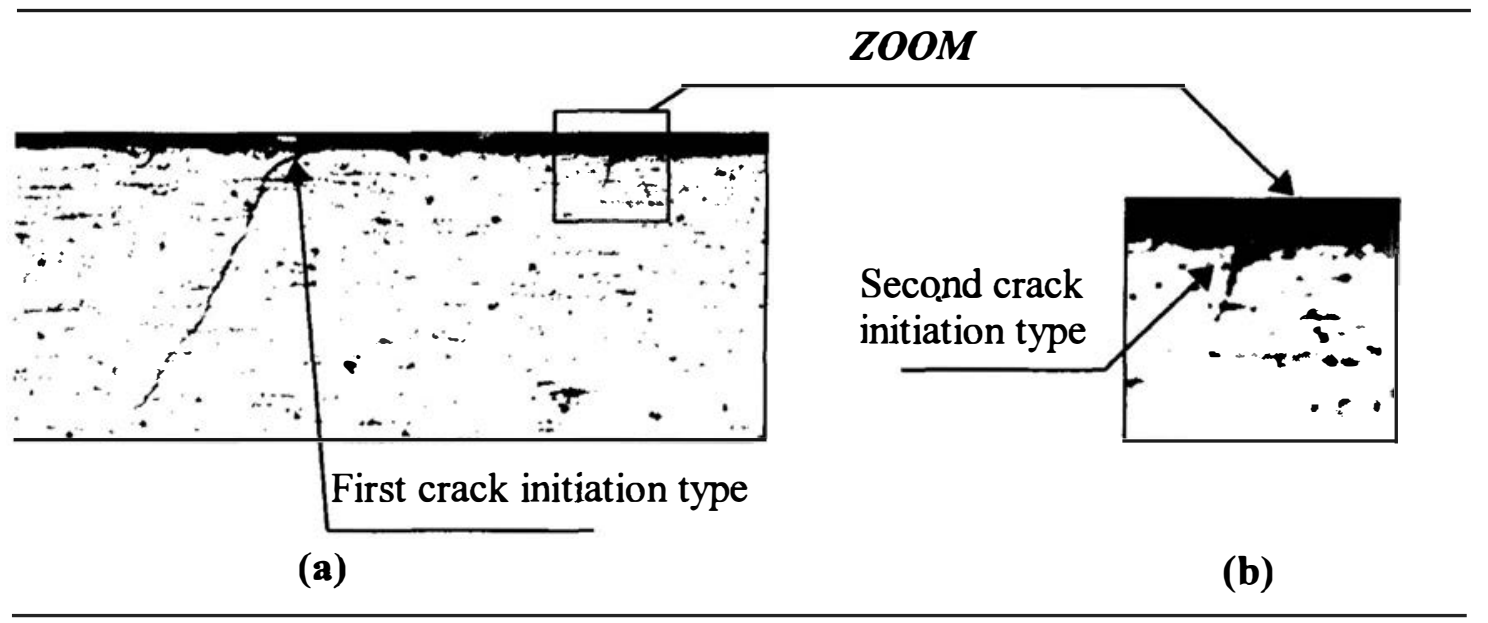

Fig. .2 Two crack initial growth directions under fretting fatigue conditions: View of specimen cross section $(0, X, Y)$. (See Fig. I for notation) 


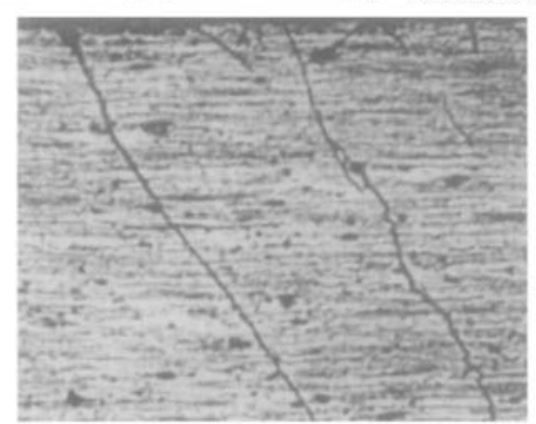

Crack network in the specimen submitted to fretting fatigue

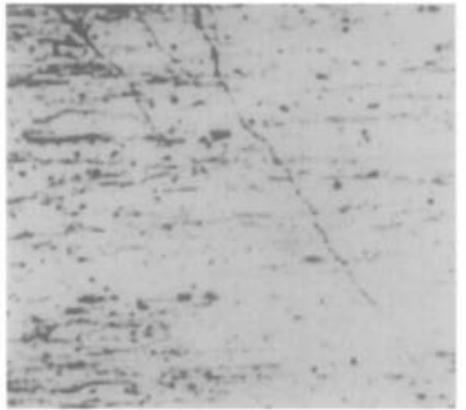

Crack network in the antagonist sphere submitted to fretting wear

Fig. 3 Crack network observed under fretting fatigue and fretting wear conditions

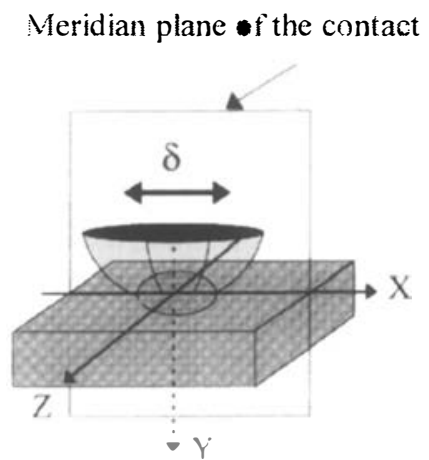

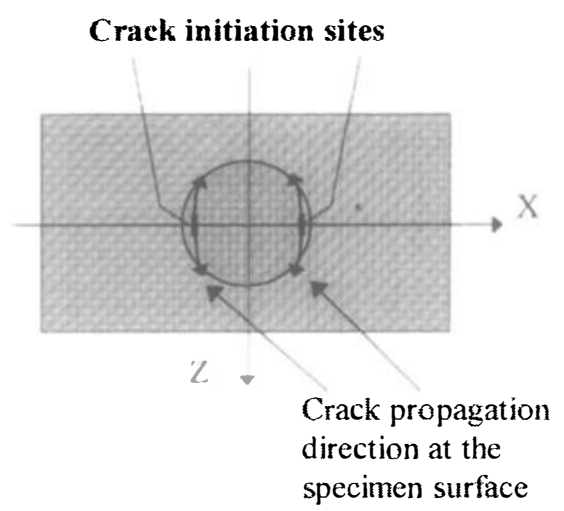

(a)

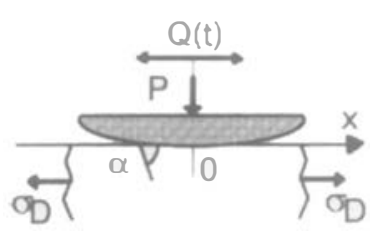

(b)

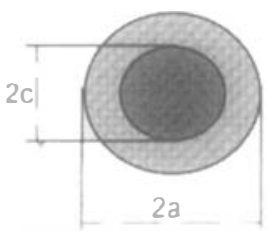

(c)

Fig. 4 (a) Definition of the meridian plane of the contact and schematic position of crack initiation sites; (b) fretting fatigue modelling; (c) contact notation

processes are generated at the middle of the contact, in the plane of imposed displacement. Furthermore the initial growth direction is also first apparent in this plane.

Thus as a starting point, a two-dimensional model is used here to calculate the continuum stress field in the meridian plane of the contact area, considering the contact surface pressure distributions in this plane.

During the experimental procedure, the flat specimen is submitted to a static traction. The spherical pad is pressed against the flat specimen and is submitted to an oscillatory displacement. This generates an oscillating tangential force at the interface of the contacting bodies. The latter are submitted to a constant normal force, $F n$, and an oscillating tangential traction, $Q(t)$ (Fig. 4(b)). The analytical results of Johnson ${ }^{10}$ are used to determine the contact area size and the partition in the stick and slip zones (Fig. 4(c)). The normal and tangential distribution of pressure are thus calculated. The stress field in the specimen is calculated by superim- posing the effect of the fretting surface loading on the external static loading, $\sigma_{D}$. In the present study, a mixed regime with a dominant stick zone is modelled. The experimental loading conditions used are summarized in Table 2.

\section{Experimental evidence}

As mentioned previously, many cracks may initiate in the fretted area. Two cracks of this network propagate Table 2 Contact conditions considered in this
study

Normal force $F n=1000 \mathrm{~N}$,

Tangential force $Q=+/-950 \mathrm{~N}$; positive from

right to left,

Global friction coefficient $\mu=1.3$,

$\Rightarrow$ Contact zone, $2 \mathrm{a}=3.57 \mathrm{~mm}$

$\Rightarrow$ Minimum stick zone, $2 \mathrm{c}=2.30 \mathrm{~mm}$ 
symmetrically to the contact centre, under the contact zone. Contrary to classical references on fretting fatigue initial growth angles, cracks were not observed to nucleate along the $45^{\circ}$ (or somewhat more) plane with respect to the component surface ${ }^{11,12}$. It must be pointed out that all the previous theoretical and experimental approaches on fretting fatigue were applied to classical fretting fatigue tests. During these tests the contact fatigue stresses were induced by bulk and surface deformations induced by a cyclic fatigue external stress (twisting, bending, tension-compression). During the tests conducted in this study, a static tension (representative for example of residual stresses) is superimposed on a fretting contact loading. This loading condition is called 'fretting fatigue static'. In the following text 'fretting fatigue' is employed instead of 'fretting fatigue static'. This new test configuration induces different crack initiation angles. Hence, two types of crack initiation angles are observed. Some cracks initiate with an angle of approximately $80^{\circ}$ to the surface near the edge of the contact. Other cracks initiate with an angle of approximately $25^{\circ}$ to the surface, in the fretted area. One or both of these two initial propagation directions was systematically observed during the fretting fatigue experiments.

The first conclusions that can be drawn from these experimental observations are:

- The crack location and initiation angles are systematically reproducible; crack initiation is not only governed by a random distribution of inhomogeneities. Initiation results from the coupling effect of cyclic loading and micromechanical phenomena.

- The fretting loading plays a major role in the crack initiation and propagation processes. Actually, the symmetrical feature of the fatigue crack network originates from the symmetrical reverse slip resulting from the symmetrical tangential traction on the surface. Furthermore, as mentioned in the first part of this study, an identical crack network was observed in the counterface which was not subjected to this static traction. This counterface being only submitted tc fretting wear indicates that analogous initiation processes take place under fretting wear. The theoretical model proposed is thus not specific to fretting fatigue problems.

- Two different types of crack angles are observed. It may be concluded that two types of crack initiation mechanisms induced by two different stressing mechanisms take place during the initiation process.

\section{Dislocation model for crack initiation}

It is well known that damage accumulation must take place to form a flaw before crack propagation takes place. Hence, any discussion of fatigue must be related to the movement of dislocations under the influence of the applied stresses. The threshold stress to cause dislocations to move is very small and therefore cycling stressing well below the elastic limit will involve the oscillation of dislocations.

The simple model for contact fatigue crack initiation proposed by Yamashita and Mura $^{8}$ is used here. Cracks are assumed to initiate by localization of plastic defor- mation. Plastic slip takes place on a weak glide plane. Dislocation loops are piled up as a result of multiple slip. The slip plane is inclined by an angle $\alpha$ with respect to the material surface as shown in Fig. 5. A schematic representation of the pile-up of dislocation loops on a slip plane is represented.

Two typical slip patterns are shown: (a) a rising pattern, (b) a sinking pattern. Figure 5(c) shows the damage accumulated at the dislocation dipoles, due to cyclic loading.

The reader should refer to Reference 8 for further explanation.

As the stress in the surface layer changes rapidly, the average value of stresses on the slip plane is used. As mentioned by Yamashita and Mura, when there is a sharp stress concentration, it is generally accepted that the absolute value of the stress at one point might not govern fatigue but some volume of material under high stress has to be considered.

In the following discussion, it is now assumed that the amplitude of the average shear stress on a plane, $\Delta \tau m$, and the maximum value of the average normal stress on the same plane, maximum of $\sigma m$, are the governing parameters for crack initiation. The average values are calculated considering an inclined plane of length $L$ (Fig. 6). $L$ must be very small in order not to introduce stresses that are not experienced very close to the material surface.

A very fine grid is defined in the general $(X, Y)$ axis. The continuum stress field is calculated over this grid. The plane inclination $\alpha$ ranges then from $0^{\circ}$ to $180^{\circ}$. The stresses are then converted in the $(n, t)$ coordinate system associated with the inclined plane to calculate the average stress along that direction. $T m$ is the average value of $\sigma n t$ and $\sigma m$ the average value of $\sigma n n$ along the plane of length $L$. In the following discussion $L$ is taken to be $20 \mu \mathrm{m}$.

\section{Results and discussion}

\section{Prediction of crack initiation angles}

The aim of this section is to identify the initiation mechanisms involving the two types of early growth stage cracks experimentally observed.

\section{First crack initiation mechanism}

Figure 7(a) shows the variation of $\sigma m$ at three different points in the contact area, depending on the inclination $\alpha$ of the initiation plane considered. It must be kept in mind that the size of the contact area in the present example is $3.57 \mathrm{~mm}$ and the size of the minimum stick zone is $2.30 \mathrm{~mm}$. The origin of the $x$-axis is at the centre of the contact zone. As can be observed, the plane inclination corresponding to $\sigma m$ maximum value varies with respect to the initiation site considered in the contact area. For example, $\sigma \mathrm{m}$ is a maximum along the $90^{\circ}$ plane for the point lying at the edge of the contact, $x=-1.785 \mathrm{~mm}$. When the point considered is in the fretted area, $x=-1.65 \mathrm{~mm}$, the maximum value of $\sigma m$ is reached for $\alpha=80^{\circ}$. At $x=$ 


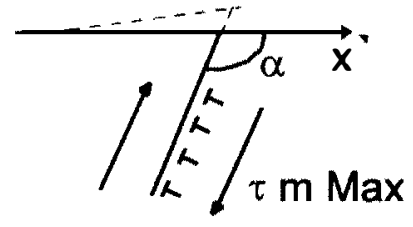

(a)

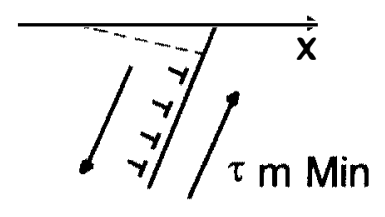

(b)

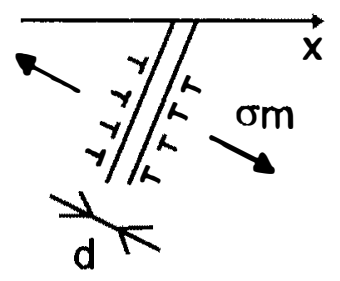

(c)

Fig. 5 Representation of the pile-up of dislocation loops on inclined plane ${ }^{8}$

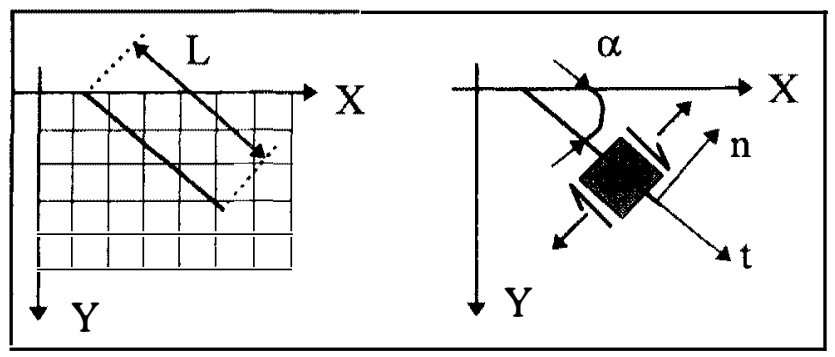

Fig. 6 Notation used for stress field calculation

$-1.45 \mathrm{~mm}$, this maximum is reached along the $75^{\circ}$ direction. Figure 7(b) shows the variation of $\sigma \mathrm{m}$ at point $x=1.75 \mathrm{~mm}$ for three stages of the fretting cycle $(\boldsymbol{\ell}=+950 \mathrm{~N}, \boldsymbol{\ell}=0 \mathrm{~N}, \boldsymbol{Q}=-950 \mathrm{~N}) \cdot \sigma \mathrm{m}$ varies between $-80 \mathrm{MPa}$ and $480 \mathrm{MPa}$ along the direction of maximum $\sigma m$. As the fretting proceeds, the volume located at this point is submitted to a tensile fatigue traction with $R \approx-0.17(R=\sigma \min / \sigma \max )$. This elementary volume is thus submitted to classical tensile fatigue. But contrary to the classical plain fatigue tests, initiation appears very early in the test life under fretting fatigue. One explanation of this early crack initiation is that the tensile stress level is quite high under fretting fatigue. Hence, an initial flaw submitted to this tensile cyclic stress may fail through a brittle tensile fracture. These remarks show that the cracks experimentally observed perpendicular to the surface are initiated through a tensile fatigue mechanism. The theoretical initiation directions linked to this tensile fatigue mechanism correlate very well with the experimental ones.

\section{Second crack initiation mechanism}

Figure 8 shows the evolution of the amplitude of $\tau m$ over a fretting cycle, $\Delta \tau m$, at three points in the contact area depending on the inclination $\boldsymbol{\alpha}$ of the initiation plane considered. The amplitude of $\tau m$ reaches a maximum value on two planes at each contact point. These planes are perpendicular to each other. The maximum-amplitude-plane inclination varies depending on the point in the contact zone. Figure 9 shows the variation of $\tau m$ and $\sigma m$ at point $x=-1.65 \mathrm{~mm}$ for two extreme values of the tangential force $(\boldsymbol{Q}=+950 \mathrm{~N}$, $=-950 \mathrm{~N}$ ), during the fretting cycle. If we consider that initiation will take place at this contact location, in the fretted area, the predicted initial crack growth direction then equals $30^{\circ}$ and $120^{\circ}$ with respect to the surface.

Experimentally, only one initial growth plane is observed at each initiation site. Here, the $120^{\circ}$ plane is not experimentally observed.

Yamashita and Mura were facing a similar problem when analysing the evolution of $\Delta \tau m$ in order to study

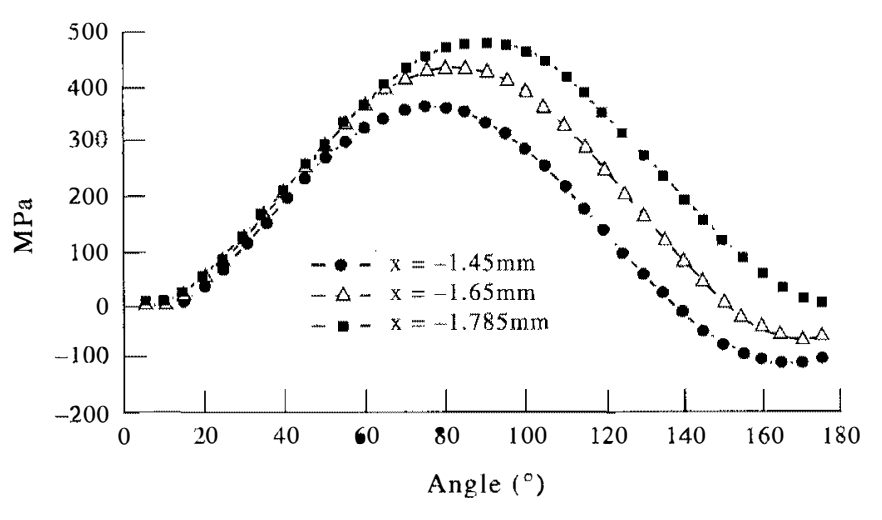

(a)

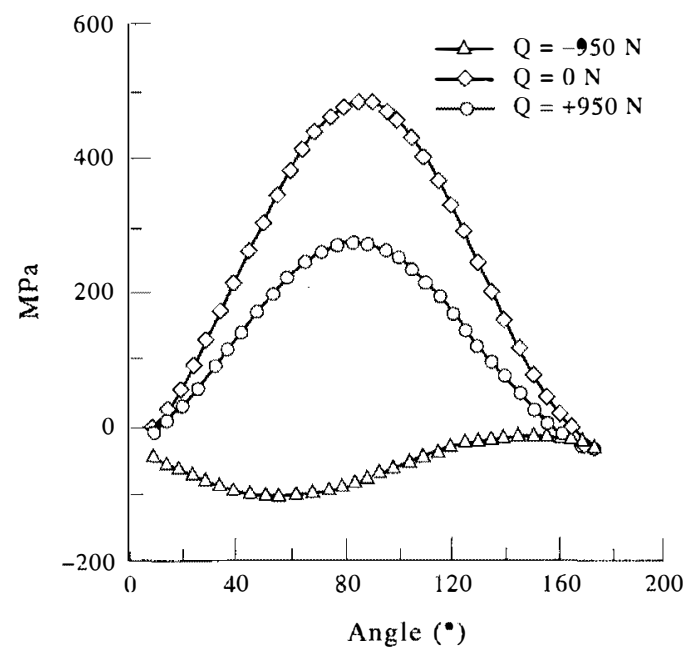

(b)

Fig. 7 (a) Evolution of om at three points in the contact area versus initiation plane inclination; (b) evolution of orm at $x=-1.75 \mathrm{~mm}$, at three loading stages 


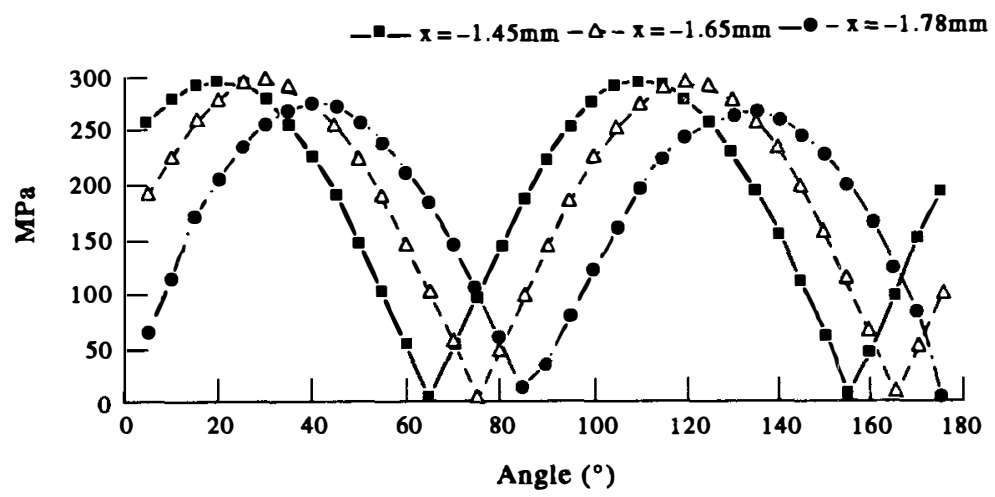

(a)

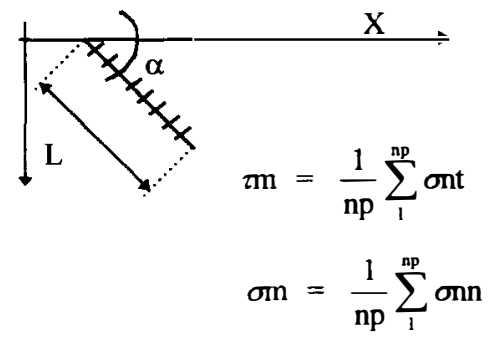

np : number of summation points on plane of length $\mathrm{L}$.

(b)

Fig. 8 (a) Evolution of $\Delta \tau m$ at three points in the contact area versus initiation plane inclination; (b) calculation of $\pi m$ and $\sigma m$

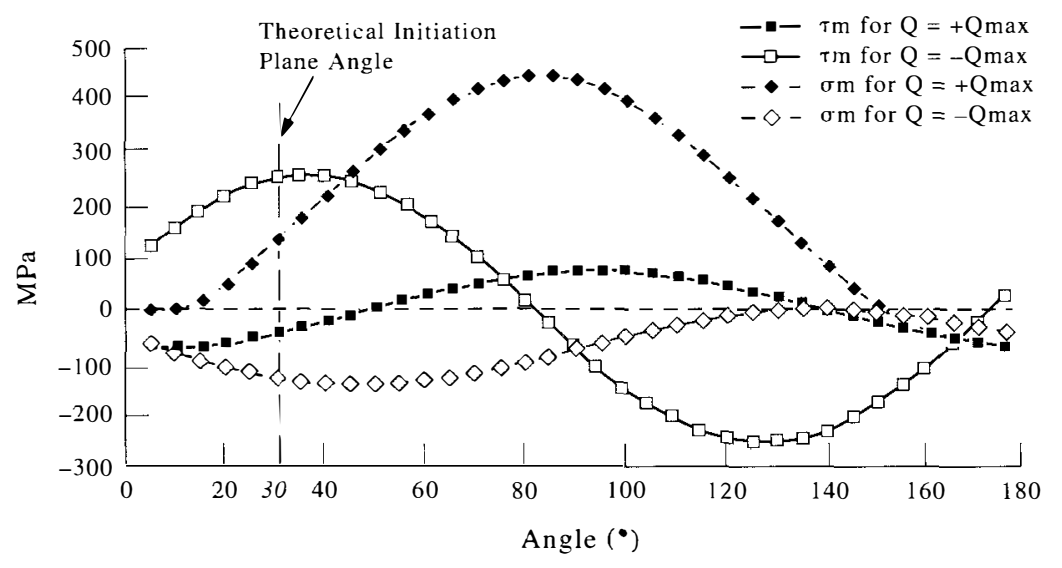

Fig. 9 Evolution of $\pi m$ and $\mathrm{om}$ at $x=-1.65 \mathrm{~mm}$, over a loading cycle, versus initiation plane inclination

the crack initiation mechanisms of pitting cracks. They applied a one-dimensional tangential force (ranging from 0 to + Qmax) and a varying normal force. They presented the evolution of $\Delta \tau m$ for three plane inclinations $\left(50^{\circ}, 90^{\circ}, 130^{\circ}\right)$ versus the abscissa of the contact points. They showed that the amplitude of the shearing stress for $\alpha=50^{\circ}$ was quite identical for $\alpha$ $=90^{\circ}$ and $\alpha=130^{\circ}$. As results on other plane inclinations were not given, it is not possible to guess if this was also encountered along other plane directions. The authors suggested that a more realistic stress analysis considering the asperity effect was necessary. So, they took into account a single asperity located at an arbitrary site in the contact in order to distinguish the evolutions of stresses along different plane inclinations.

This solution is not considered here. In the authors' opirion, it is not a statement of the obvious to consider the influence of a single asperity in order to study the asperity effect whereas multiple asperities may act as metallic junctions in the contact area. So, two other explanations are proposed to explain the 'non-initiation' alorg this direction.

First, it has to be noted that the $\Delta \tau m$ value along the $120^{\circ}$ plane is slightly smaller than this quantity along the $30^{\circ}$ plane. But the difference (respectively $288 \mathrm{MPa}$ on the $120^{\circ}$ plane and $294 \mathrm{MPa}$ on the $30^{\circ}$ plane) is too small to give a physical explanation.

Secondly, the $\sigma m$ values experienced along these two $\left.\Delta \tau m\right|_{\text {maximum }}$ planes vary differently during a fretting cycle. Along the $120^{\circ}$ plane, $\sigma m$ varies from $-11 \mathrm{MPa}$ to $250 \mathrm{MPa}$. Along the other direction $\left(30^{\circ}\right)$, the $\sigma \mathrm{m}$ value ranges from $-127 \mathrm{MPa}$ to $128 \mathrm{MPa}$ (Fig. 9). Mura and Nakasone ${ }^{5}$ in presenting their vacancydislocation-dipole-model pointed out the importance of the distance $d$ (Fig. 5) between the two dislocation layers along the slip direction. These two layers have to be close enough to be able to stimulate reverse slip on each other. When the layers are far from each other or too close, this stimulation or acceleration cannot be seen. This can explain the inefficiency of the $120^{\circ}$ plane to nucleate a crack. Actually, the $\sigma m$ values experienced along this plane are very high and not symmetrical around $\sigma m=0$. Hence, $\sigma m$ will tend to move apart the dislocation layers at each fretting cycle and thus annihilate their interaction. On the other hand, $\sigma m$ along the $30^{\circ}$ plane is totally symmetrical around $\sigma m=0$. Hence, the distance $d$ between the dislocation layers oscillates between a constant value, enabling reverse slip stimulation from one layer on the other. The model gives here a physical explanation to the non-initiation of a crack along the $120^{\circ}$ plane that was 
experimentally observed. The influence of shear stresses to enable pile up of dislocations and dislocation motion, but also the influence of $\sigma \mathrm{m}$ to stimulate slip along dislocation layers, have to be taken into consideration.

Hence, at each contact point, two initiation planes are first theoretically predicted through the maximum value of $\Delta \tau m$. But one of these two planes is not physically acceptable due to a major reason explained previously. In Fig. 9, the evolution of $\sigma m$ and $\tau m$, at point $x=$ $-1.65 \mathrm{~mm}$, for $Q=+1-950 \mathrm{~N}$, is represented versus initiation plane inclination. Along the plane $\alpha=30^{\circ}$ corresponding to $\Delta \tau m$ maximum, dislocations are submitted to reciprocating shear stress and tension compression. Reverse slip thus proceeds all along a fretting cycle along this initial growth direction. Hence, a process of rising and sinking slip takes place. This process was already described as an extrusionintrusion mechanism.

Chivers et al. ${ }^{13}$ proposed palliatives to fretting fatigue damage and thus proposed to reduce the tensile traction at the surface. The present theoretical results demonstrate that crack initiation is also induced by shear stresses along specific planes. Hence, it may be erroneous to try to reduce fretting damage only by considering tensile stress reduction.

At this stage of the study, two initiation mechanisms have been identified to explain the two major crack initial growth directions experimentally observed under fretting fatigue loading. The next step of this approach is to link each of these mechanisms to a parameter predicting the initiation site.

\section{Location of crack initiation}

\section{Location of tensile fatigue cracks}

Figure 10(a) shows the evolution of the maximum value of $\sigma m$ versus the potential crack location in the contact area. The corresponding crack initiation angles linked to this maximum value are also reported. Positions of contact points are recalled in Fig. 10(b). As mentioned previously, a high tensile stress level is necessary to initiate a crack along these directions. $\sigma m$ increases very rapidly at the trailing edge of the contact zone. Hence, tensile fatigue cracks initiate very close to the edge of the contact, inside or outside this area. The corresponding crack initiation directions range from $90^{\circ}$ (outside the contact) to $75^{\circ}$ (inside the contact). These crack inclinations and locations correlate very well the experimental observations.

It is very interesting to note that cracks are also predicted to nucleate outside the contact area via a brittle tensile fracture mechanism. Contrary to Ruiz's parameters that predict no crack initiation outside the contact area, this model gives 'sharper' answers to crack initiation under fretting fatigue loading.

\section{Location of shear cracks}

As demonstrated before, the parameters governing crack initiation induced by an extrusion-intrusion process are the amplitude of $\tau m$ and the variation of $\sigma m$ along that direction. In Fig. 11(a), the evolution of the maximum values of $\Delta \tau m$ over a fretting cycle versus the abscissa of contact points is represented (diamond shape points). On the same figure, the evolution of the mean value, $\sigma a$, of the tensile traction $\sigma m$ along the theoretical initiation direction is reported versus the abscissa of the contact points (black points). The contact dimensions are recalled in Fig. 11(c). A schematic definition of $\sigma a$ is also presented in Fig. 11(b): $\sigma m$ oscillates around $\sigma a$ during a fretting cycle.

The variation of $\Delta \tau m$ is very small in the fretted contact area. Hence, the location of the cracks initiated through shearing stresses cannot be predicted only by the variation of $\Delta \tau m$. The variation of $\sigma m$ via the evolution of its mean value $\sigma a$ is then incorporated for the prediction of these initiation loci.

A first zone is defined in Fig. 11(a), the hashed zone including abscissa $-1.10 \mathrm{~mm}<x<-1.35 \mathrm{~mm}$. At

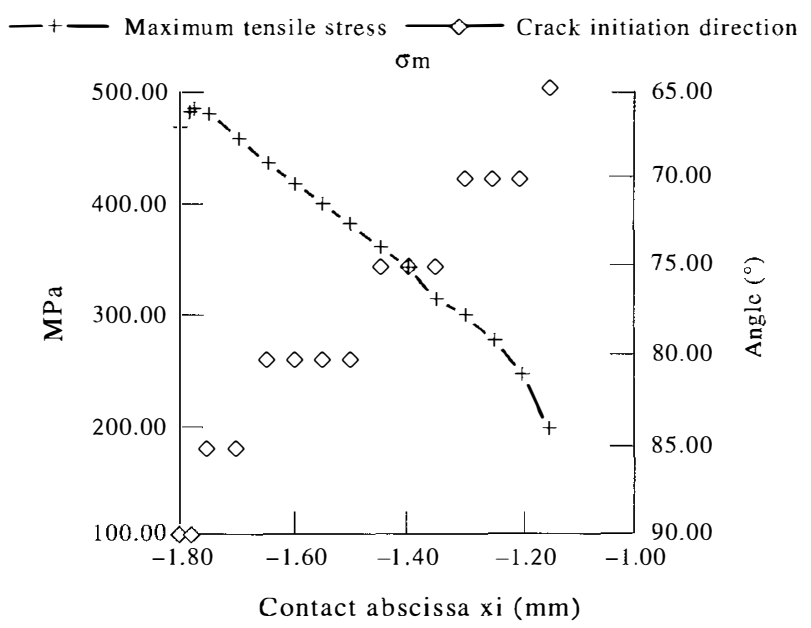

(a)

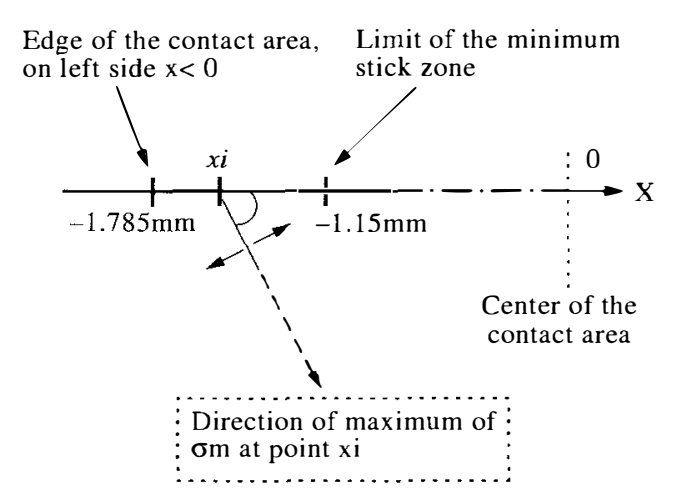

(b)

Fig. 10 (a) Evolution of the maximum value of om versus contact points and corresponding initial growth angles; (b) definition of contact points plotted 


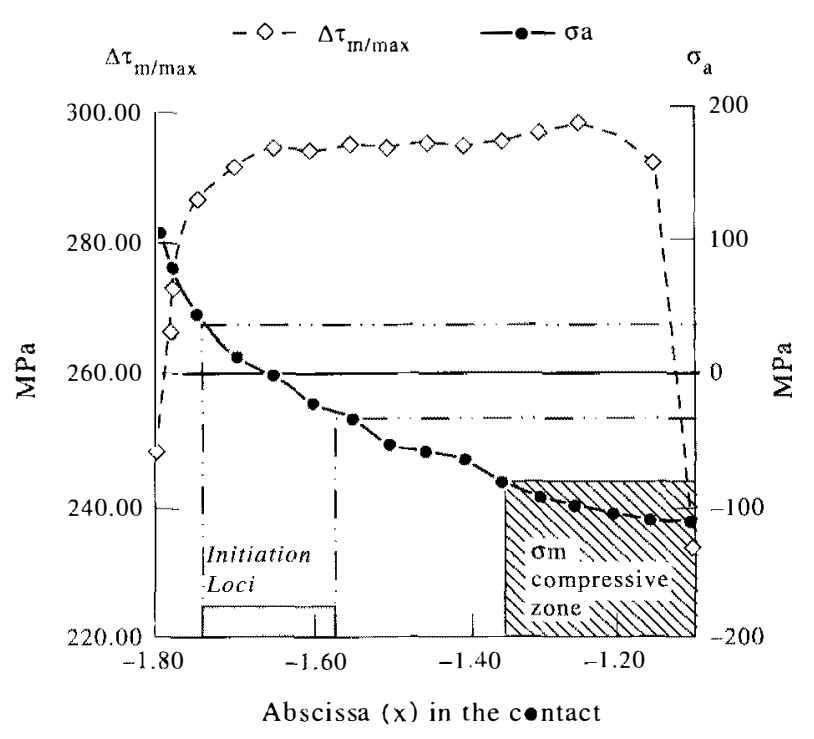

(a)

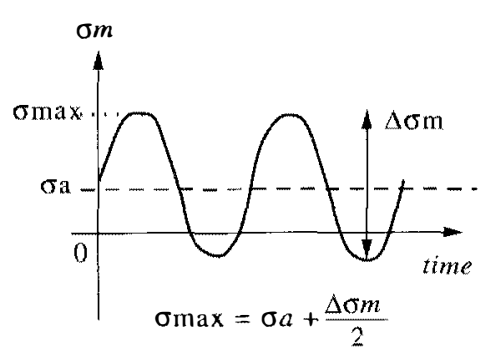

(b)

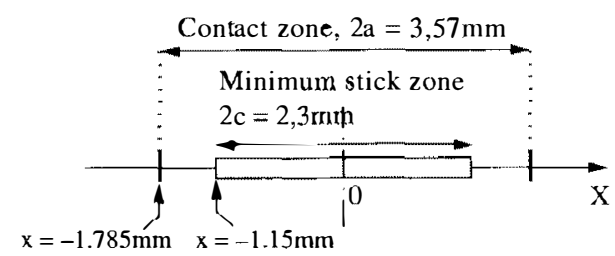

(c)

Fig. 11 (a) Evolution of the maximum value of $\Delta \pi m$ and variation of $\sigma a$ versus contact point abscissa; (b) definition of $\mathrm{\sigma a} ;$; (c) dimensions of some contact zones

these contact points, the material elements along the precicted initial growth direction are in a compressive state all along a cycle, $\sigma a$ is less than $-75 \mathrm{MPa}$. For example at $x=-1.20 \mathrm{~mm},-134 \mathrm{MPa}<\sigma m<$ $-83 \mathrm{MPa}$. When the contact point reaches the edge of the contact, $\sigma a$ tends to $100 \mathrm{MPa}$. For example, for $x$ $=-1.78 \mathrm{~mm},-56 \mathrm{MPa}<\sigma m<256 \mathrm{MPa}, \sigma a$ equals $100 \mathrm{MPa}$. On these two defined contact zones, the absolute value of $\sigma a,|\sigma a|$, is high. This means that $\sigma m$ ranges between either two high tensile values or two high compressive values, along the predicted crack initial growth directions. In both cases, crack initiation will not be stimulated, the dislocation layers being respectively too far or too close from each other. Reverse sliding along each dislocation layer is then graciually annihilated.

On the other hand, when $\sigma m$ is quite symmetrical around $\sigma m=0$, that is when $\sigma a$ approximately equals zer, the reverse slip along the dislocation layer will result from the coupling action of $\tau m$ and $\sigma m$ along that direction. As observed in Fig. 11(a), this condition is obtained for $-1.75 \mathrm{~mm}<x<-1.55 \mathrm{~mm}$. Furthermore for $x=-1.65 \mathrm{~mm}, \sigma a$ equals zero. This is the condition under which the dislocation stimulation is the more efficient along the dislocation layers. Thus potential initiation loci are predicted through the variation of $\Delta \tau m$ and the mean value of $\sigma m$ along crack initiation directions. Crack initiation induced by an extrusion-intrusion mechanism is shown to occur in the fretted area, neither too close to the trailing edge nor too close to the minimum stick zone of the contact. This crack initiation domain is experimentally observed, many cracks being initiated in this area.

\section{Crack interaction leading to spall detachment}

Spall detachment is a common wear mechanism encountered under fretting fatigue conditions. This damage may be induced by complicated processes such as microstructural transformation (second phase), presence of high strains, plasticity etc. Spall detachment may also occur by coalescence of microcracks.

As demonstrated previously, many cracks may initiate in a very small area and along different directions. Thus these microcracks may meet each other. A schematic representation of this phenomenon is reported in Fig. 12. One crack is initiated by one of the two mechanisms identified previously. Another crack is initiated either by the same mechanism or by the other one in the neighbourhood of the first crack. As these cracks are not initiated through the same process or are not located at the same site, they will propagate along different directions. Thus they may join each other leading to the detachment of the central part surrounded by these two cracks. This phenomenon is observed in Fig. 2(b). Two cracks were initiated close to each other in the right side of the contact $(x>0)$. A crack was initiated by a tensile fatigue mechanism. On its right a second crack was initiated through an extrusion-intrusion mechanism, at a more oblique angle. As can be observed the coalescence of these cracks led to the detachment of a small surface particle.

Near the stick contact area, cracks initiated through maximum shear stresses are inclined along a near-zero direction (Fig. 12). These cracks may exist in a very dense network and their inclination may also lead to particle detachment.

\section{Conclusions}

This simple dislocation model allows identification of two initiation mechanisms existing during fretting fatigue cracking. Through a surface layer stress analysis, cracks were shown to initially grow:

- Along a direction ranging from $80^{\circ}$ to $90^{\circ}$ with respect to the surface through a brittle tensile frac- 


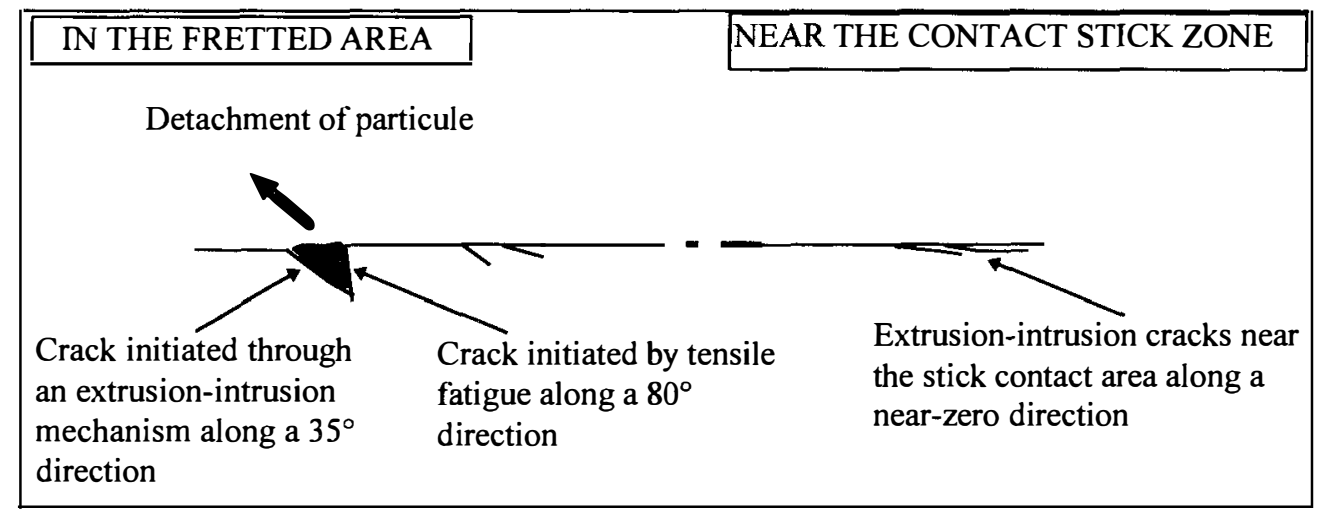

Fig. 12 A simple mechanism for spall detachment

ture mechanism governed by traction stresses along that direction. This initial growth plane depends on the crack location in the contact area.

- Along a direction ranging from $25^{\circ}$ to $35^{\circ}$ with respect to the surface through a reverse slip mechanism. Sliding originates from dislocation movements along persistent slip bands. Alternative reverse slip and traction-compression act on this initiation planes. Resulting from this cyclic sliding, a process of extrusion and intrusion on slip bands is thus predicted. This mechanism is governed by the shear stresses acting on the dislocation layers that will lead to the formation of a crack. Furthermore, the distance between the two dislocation layers is taken into consideration via the analysis of the tensile stress along the initial growth plane.

This new approach coupling the effect of sliding on dislocation layers and of the distance between these layers gives a physical explanation to the experimental initiation angles observed. It correlates very well with the experimental initial propagation directions.

Two parameters are proposed to predict crack location with regards to these initiation mechanisms. Each of these parameters is linked to one of the two initiation mechanisms proposed previously. The tensile fatigue cracks nucleate very close to the contact edge, inside or outside the contact area. Considering the coupling effect of the shear stresses and tensile stresses along each initiation direction, cracks formed by an extrusion-intrusion mechanism are shown to occur in the fretted area, in a specific domain. These theoretical results correlate very well with the experimental observations on the location of cracks initiated under fretting fatigue loading. This theoretical model tested under fretting fatigue conditions is also applicable to fretting wear cracking problems. During the experiments, similar crack networks were observed on the specimen only submitted to fretting wear. This theoretical approach is, a priori, not specific to fretting fatigue damage.

With regards to these initiation processes, a spall detachment mechanism is proposed considering the interaction of microcracks initiated along different directions.

\section{References}

1. Dang Van K., Griveau B. and Message O. On a new multiaxial fatigue limit criterion: Theory and applications. In Biaxial and Multiaxial Fatigue. (Ed. M.W. Brown). Mechanical Engineering Publications, London, 1989, 479-496

2. Ruiz C., Boddington P.H.B. and Chen K.C. An investigation of fatigue and fretting in a dovetail joint. Exp. Mech., 1984, 208-217

3. Hills D.A. and Nowell D. Mechanics of Fretting Fatigue. Kluwer Academic, Dordrecht, 1994, 232

4. Lamacq V., Dubourg M.-C. and Vincent L. Crack path prediction under fretting fatigue, a theoretical and experimental approach. ASME, J. Tribol. 1996, 118, 711-720

5. Mura T. and Nakasone Y. A theory of fatigue crack initiation in solids. J. Appl. Mech., 1990, 57, 1-6

6. Cheng W., Cheng H.S., Mura T. and Keer L.M. Micromechanics modeling of crack initiation under contact fatigue. ASME Trans., 1994, 116, 2-8

7. Nowell D. and Hills D.A. An analysis of fretting fatigue. Proc. Instn. Mech. Engrs., 1987, 965-973

8. Yamashita N. and Mura T. Contact fatigue crack initiation under repeated oblique force. Wear 1983, 91, 235-250

9. Zhou Z.R. and Vincent L. Effect of external loading on maps of aluminium alloys. Wear 1993, 162-164, 619-623

10. Johnson K.L.. Contact Mechanics, Cambridge University Press, 1985, 451

11. Alic J.A. and Hawley A.L. On the early growth of fretting fatigue cracks. Wear 1979, 56, 377-389

12. Waterhouse R.B. Fretting Fatigue, Applied Science, London. 1981

13. Chivers T.C. and Gordelier S.C., Fretting fatigue and contact conditions: A rational explanation of palliative behaviour. Proc. Instn. Mech. Engrs, 1985, 199, 325-337 